\title{
Characterisation of the convective heat transfer from the surface of a TGV brake disc in the actual environment using infrared thermography
}

\author{
by M. Siroux, S. Harmand, B. Desmet \\ Laboratoire de Mécanique et Energétique, Université de Valenciennes et du Hainaut Cambrésis, Le \\ Mont Houy, 59313 Valenciennes Cedex 9
}

\begin{abstract}
:
This paper presents an experimental technique wich allows to reach the local convective heat transfer coefficient on a rotating TGV brake disc model in the actual environment and submitted to an air flow parallel to the disc surface. The heat transfer measurement technique is based on the combination of infrared thermography and of a numerical computation code. Experimental set-up, infrared temperature determination and results are detailed.
\end{abstract}

\section{Introduction}

The TGV train high energy braking from $320 \mathrm{~km} / \mathrm{h}$ to 0 involves a significant brake disc temperature increase (local temperatures ranging between $500-1000{ }^{\circ} \mathrm{C}$ ) and, consequently, it is necessary to optimise the disc cooling in order to maintain the thermal stress at an acceptable level. The air flow under the carriages produced by the train displacement is not very well known. Actually, the air flow velocity around the brake disc seems to be rather small and a specific design to direct the air stream towards the disc should be a solution of interest to improve the disc cooling.

The aim of the present work is the experimental determination of the local convective heat transfer coefficient on a rotating TGV brake disc model in the actual environment and submitted to an air flow parallel to the disc surface. The heat transfer on a rotating disc in still air was the subject of many works. Von Karman [1] showed that laminar, transition and turbulent flows exists on a rotating disc. Gregory, Stuart and Walker [2] highlighted two critical radius $\left(R_{c}\right.$ and $\left.R_{t}\right)$ and two rotational Reynolds numbers $\left(R e_{c}=\omega R_{c}^{2} / v_{a}=1.82 \times 10^{5}\right.$ and $R e_{t}=\omega R_{t}^{2} / v_{a}=2.82 \times 10^{5}$ ) on the disc which separates the three flow modes. In the central zone to the radius $R_{C}$ the flow is purely laminar, and beyond the radial position $R_{T}$ this one is entirely turbulent. Dorfman [3] proposes a correlation giving the local Nusselt number as function as rotational Reynolds number. The bibliography is poorer with regard to the study of the heat transfer on the rotating disc submitted to an air flow. De Vere [4] proposes a correlation when speeds of transverse flow and rotation of the disc are connected by the relation $U=\omega R$. De Vere stresses the fact that the heat transfer on the disc is dominated by the principal flow of air more than by the secondary rotational flow.

\section{Principle of the method}

The experimental method is based on the use of a thermally thick wall combined with the technique of temperature measurement by infrared thermography and with the numerical solution of the steady state heat equation. The thermally thick wall is a steel disc covered with a ceramic coating. The thermally insulating disc is heated by electric resistances on one face and cooled on the other one by an air flow (Figure 1). Through the low conductivity of ceramics, the variations of the convective heat transfer coefficients $h$ along the disc radius $r$ are translated into a variation of surface temperature along the radius which can be detected.

The local convective heat transfer coefficient $h$ is obtained from the numerical solution of the steady state heat conduction equation inside the ceramic part of the disc : 


$$
\frac{\partial^{2} \mathrm{~T}}{\partial \mathrm{r}^{2}}+\frac{1}{\mathrm{r}} \frac{\partial \mathrm{T}}{\partial \mathrm{r}}+\frac{\partial^{2} \mathrm{~T}}{\partial \mathrm{z}^{2}}=0
$$

The known boundary conditions of the problem are : for $z=0, T(r, 0)$ is given by the infrared thermography and for $z=e, T(r, e)$ is given by the thermocouples. To solve these equations, we used a finished differences method.

The local Nusselt number is deduced of the heat thermal balance on the disc surface :

$$
\mathrm{Nu}=\frac{h_{\mathrm{r} . r}}{\lambda_{\mathrm{a}}}=\frac{\lambda_{\mathrm{c}}\left(\frac{\partial \mathrm{T}}{\partial \mathrm{z}}\right)_{z=0}-\frac{\varepsilon}{1-\varepsilon}\left(\sigma \mathrm{T}^{4}(r, 0)-J\right)}{\mathrm{T}(\mathrm{r}, 0)-\mathrm{T}_{\infty}} \cdot \frac{\mathrm{r}}{\lambda_{\mathrm{a}}}
$$

with :

$-\lambda_{\mathrm{a}}, \lambda_{\mathrm{c}}$

$-\left(\frac{\partial T}{\partial z}\right)_{z=0}$

air and ceramic conductivitys $\left(\mathrm{W} \cdot \mathrm{m}^{-1} \cdot \mathrm{K}^{-1}\right)$

$-\varepsilon$

surface temperature variation (W. $\mathrm{m}^{-1}$ )

$-\sigma=5.67 \times 10^{-8}$

disc emissivity

- $\mathrm{T}(\mathrm{r}, 0)$

constant of Stefan $\left(\mathrm{W} . \mathrm{m}^{-2} \cdot \mathrm{K}^{-4}\right)$

$-\mathrm{J}$

disc surface temperature $(\mathrm{K})$

disc radiosity $(\mathrm{W})$.

The surface temperature variation $\left(\frac{\partial T}{\partial z}\right)_{z=0}$ is obtained by the numerical solution of the heat equation, the disc surface temperature $T(r, 0)$ and the disc emissivity $\varepsilon$ are obtained by infrared thermography, the temperature of the flow $T_{\infty}$ is measured by thermocouple and the disc radiosity $\mathrm{J}$ is deduced from the radiatives exchanges between the disc surface and the surface of observation disc [5].

$$
\mathrm{J}=\frac{\varepsilon \sigma \mathrm{T}(\mathrm{r}, 0)^{4}+(1-\varepsilon) \varepsilon_{\mathrm{s}} \sigma \mathrm{T}_{\mathrm{S}}^{4}}{1-(1-\varepsilon)\left(1-\varepsilon_{\mathrm{S}}\right)}
$$

with $\varepsilon_{\mathrm{S}}=0.65$ and $T_{\mathrm{S}}$ are respectively the emissivity and the temperature of the observation disc in aluminium.

\section{Experimental study}

\subsection{The experimental device}

The test rig (scale 0.617) is composed of a model disc with all the representative parts of the actual TGV brake system (flanges, pads, brake lining holders, cylinder and brake rods) (Figure 2, Figure 3). The disc is of steel (inner radius $R_{1}=51 \mathrm{~mm}$, outer radius $R_{2}=197$ $\mathrm{mm}$, thickness $\mathrm{e}^{\prime}=26 \mathrm{~mm}$ ) covered on one face and the side faces of a ceramic coating by plasma projection (conductivity $\lambda_{c}=0.65 \mathrm{~W} \cdot \mathrm{m}^{-1} \mathrm{~K}^{-1}$, thickness $\mathrm{e}=1.7 \mathrm{~mm}$ ). The thickness of the low conductivity ceramics layer has been determined in a way to obtain significant disc surface temperature variations corresponding to the surface heat transfer coefficient distribution. Eight electric resistances drowned in the steel part of the disc allows to simulate the energy dissipation by friction. The disc and its actual environment are inside the test section (dimensions $1037 \times 671 \times 225$ ). One of the walls of the aluminium casing is equipped with a observation disc (diameter $470 \mathrm{~mm}$ ) which comprises 2 slits of dimensions $107 \times 13$ $\mathrm{mm}$ making it possible to visualize the surface temperature by infrared thermography.

The measured parameters are the disc temperature surface, the temperature at the interface ceramic/steel, the temperature of the flow, the rotational speed and the air flow velocity. The disc temperature surface is determinated by infrared thermography (cf $\S 3.2$.) The temperature at the ceramic/steel interface is measured by 2 thermocouples respectively placed at 70 and $170 \mathrm{~mm}$ of the centre of the disc and the absolute error $\Delta T$ on this 
temperature is $\Delta T= \pm 0.3 \mathrm{~K}$. The temperature of the flow $T_{\infty}$ is measured by a thermocouple placed inside the test section and the absolute error absolute $\Delta T_{\infty}$ on $T_{\infty}$ can be estimated at $\Delta \mathrm{T}_{\infty}= \pm 0.3 \mathrm{~K}$. The rotational disc speed can be varied from 300 to $2000 \mathrm{rpm}$ using an engine at variable speed and the relative error made on the rotational speed measurement is $0.5 \%$. The air flow velocity can be varied from 0 to $12 \mathrm{~m} / \mathrm{s}$ using a blower. The value and the direction the air flow velocity are standardized thanks to a honeycomb. The air velocity is measured using a Pitot tube placed in the test section casing. The tests are carried out for 3 different air flow velocities $U_{1}=3 \mathrm{~m} . \mathrm{s}^{-1}, U_{2}=6 \mathrm{~m} \mathrm{~s}^{-1}$ and $U_{3}=12 \mathrm{~m} \mathrm{~s}^{-1}$. The range of air flow velocities studied here is relatively weak compared to speed of the TGV. This is due to the study objective which consists to show the improvement of the TGV brake disc cooling, avoiding the high braking temperatures, by channelling the air stream towards the disc.

\subsection{Temperature measurements and infrared system calibration}

A short wave infrared thermography camera (AGEMA $900 \mathrm{SW} / \mathrm{TE}$ ) in large band version $(2-5.4 \mu \mathrm{m})$ has been used to determine the brake disc surface temperature. The rotation of the disc imposes the use of the line scanning mode, which has a rather high frequency of the line acquisition $(f=3472 \mathrm{~Hz})$. The camera is installed in front of the disc so that its optical axis coincides with the disc rotational axis and the temperature distribution is obtained along a radius of the disc surface (Figure 1 ).

The determination of the surface temperature by infrared thermography passes by the measurement of the sample radiation [6]. In the mean time, the radiation receveid by the scanner detector consists of the thermal disc radiation attenuated by the atmosphere, the reflected radiation from surroundings attenuated by the atmosphere and the radiation emitted from the atmosphere itself. In order to minimise the undesirable effects of reflected flux and to emphasise the thermal disc emission, the disc surface has been painted with a high emissivity black paint. The black paint is supposed to be an opaque, lambertian and grey surface in emission and reflexion.

The thermal value for the measured total radiation is :

$$
\mathrm{I}_{\mathrm{m}}=\tau . \varepsilon . \mathrm{I}(\mathrm{T})+\tau .(1-\varepsilon) \mathrm{I}\left(\mathrm{T}_{\mathrm{env}}\right)+(1-\tau) \mathrm{I}\left(\mathrm{T}_{\mathrm{atm}}\right)
$$

with :

$-\tau \quad$ atmospheric transmission

- I(T) thermal value of the disc covered of black painting

$-I\left(T_{\text {env }}\right) \quad$ thermal value of the environment

$-I\left(T_{a t m}\right) \quad$ thermal value of the atmosphere

The infrared camera calibration allows to obtain the calibration curve, which connects black body thermal value I(T) and temperature T. The black body is an AGEMA BB 400-3 of emissivity equal to $0,99 \pm 0,01$. Its temperature is given by the manufacturer as being $\pm(0,3+0,004 \mathrm{~T})$.

The black paint calibration leads to the determination of the black paint emissivity in the spectral band of the infrared camera for several different temperatures. Two measurements of I are carried : one on the black paint disc surface (giving $I_{m}$ ) and the other one on a lambertian aluminium surface (giving $\mathrm{l}_{\mathrm{a}}$ ) :

$$
\mathrm{I}_{\mathrm{a}}=\tau\left|\left(\mathrm{T}_{\text {env }}\right)+(1-\tau)\right|\left(\mathrm{T}_{\mathrm{atm}}\right)
$$

The black paint emissivity is done using (4) and (5) :

$$
\varepsilon(T)=\left(I_{m}-I_{a}\right) /\left(\tau\left|(T)-I_{a}+(1-\tau)\right|\left(T_{\text {atm }}\right)\right)
$$

The disc temperature surface $T$ and the atmospheric temperature $T_{\text {atm }}$ are measured by thermocouples. So, the calibration curve allows the determination of the thermal values I(T) 
and $\mathrm{I}\left(\mathrm{T}_{\mathrm{atm}}\right)$. It is then possible to reach the black paint emissivity. Figure 4 shows the emissivity values obtained for several different temperatures. It is noticed that for a temperature ranging between $30^{\circ} \mathrm{C}$ and $80^{\circ} \mathrm{C}$ (which is temperature range used for the tests), the black paint emissivity are $\varepsilon=0.93 \pm 0.02$.

After calibrations, I $\left(T_{\text {env }}\right)$ is determined by infrared thermography. The equation (4) enables us to deduce the disc thermal value $\mathrm{I}(\mathrm{T})$. Using the calibration curve we obtain then disc temperature surface. The uncertainty obtained on the disc temperature $T$ is $\Delta T= \pm 0.90$ $\mathrm{K}$.

\section{Results and discussion}

The test were carried out for rotational speed between $\omega=325$ and $2000 \mathrm{rpm}$ ( $88500 \leq$ Re $\leq 545000$ ) which enables us to have laminar and turbulent flow on the disc, and for air flow velocity ranging between 0 and $12 \mathrm{~m} \cdot \mathrm{s}^{-1}\left(0 \leq \mathrm{Re}_{0} \leq 153000\right)$.

Figure 5 shows the contribution of the radiative losses compared to the convective losses. In the case of a rotating disc in still air (Figure $5 \mathrm{a}, \mathrm{Re}_{0}=0$ ) for weak rotational speed value $(R e=88500)$ the radiative losses are very significant (on average $44 \%$ ). It is thus necessary to take them into account in the calculation of the heat transfer coefficient. However, for a high rotational speed these losses decrease considerably (they are about $7 \%$ ). In the case of a rotating disc in air flow (Figure $5 b, R_{0}=153000$ ) the radiative losses are very weak (in order of $5 \%$ ) and thus it is not necessary to take them into account in the calculation of the heat transfer coefficient.

Figures 6 presents the variation of the local Nusselt number as a fonction of dimensionless disc radius $r^{*}=r-R_{1} /\left(R_{2}-R_{1}\right)$. For a rotating disc in still air (Figure 6a, $R e_{0}$ $=0$ ), the convective exchange increases significantly with the rotational speed. The peripheral Nusselt number increases by $650 \%$ when the rotational Reynolds number varies from 88500 to 545000 . Our results have been compared to the correlations proposed by Dorfmann. For a weak dimensionless disc radius $r^{*}<0,5$, corresponding to the with of the brake shoe, one notices good correlations. Beyond $r^{*}=0,5$, our resulted are higher than the predictions of Dorfman. For a rotating disc in air flow (fig $6 \mathrm{~b} \mathrm{Re}_{0}=153000$ ), the heat transfer increase is less significant than in the case of a rotating disc in still air. Thus, the peripheral Nusselt number increases by $39 \%$ when rotational Reynolds number increases from 88500 to 545000 . If one compares the figures $6 \mathrm{a}$ and $6 \mathrm{~b}$, one notices that for a weak rotational speed value $(R e=88500)$ convective exchange is dominated by the air flow. The peripheral Nusselt number increases by $520 \%$ at the time of the passage of $\operatorname{Re}_{0}=0$ with $\operatorname{Re}_{0}=$ 153000. On the other hand, for a high rotational speed $(R e=545000)$ the convective exchange is dominated by the rotation. The peripheral Nusselt number increases by $56 \%$ at the time of the passage of $\operatorname{Re}_{0}=0$ with $\mathrm{Re}_{0}=153000$.

A last comparison is obtained between our results and a correlation given by De Vere [4] when rotational speed and air flow velocity are connected by the equality $U=R . \omega$ (Figure 7). For a weak dimensionless disc radius $r^{*}<0.4$ our test results are close to those given by De Vere. A contrario, for significant dimensionless disc radius $r^{*}>0.4$ our values are much higher than those of De Vere. This difference can be explained by the influence of the TGV disc actual environment (our experimental configuration comprises the TGV disc actual environment, which is not the case for the study undertaken by De Vere) and by the nature of temperature distribution (the tests of De Vere are carried out on an isothermal disc, whereas in our case the disc temperature distribution approaches a in power law $T-T_{\infty}=c . r^{n}$ ).

\section{Conclusion}

The local convective heat transfer coefficient on a TGV brake disc placed in its actual environment is experimentally obtained for the rotational speed making it possible to have laminar and turbulent flow on the disc. The comparison of our experimental results, concerning the local convective exchanges on the disc, with the ones of the literature, 
allowed a significant increase in heat transfer in comparison with the case of a single disc. These results allows the influence of the TGV disc actual environment and of the nature of the temperature distribution law on its surface on the heat transfer to be shown.

\section{References}

[1] VON KARMAN, T., Über laminare und turbulente Reibung, Z. Angew. Math. Mech 1, 1921, p 244-252.

[2] GREGORY N., STUART J. T., WALKER W. S., On the stability of three dimensional boundary layers with application to the flow due to a rotating disk, Phil. Trans. Roy. Soc. London A248, 1955, p 155-199.

[3] DORFMAN L.A, Hydrodynamic resistance and heat loss from rotating solids, Oliver and Boyd, Edinburgh and London, 1963.

[4] DE VERE A.P.C, Convective heat transfer from a rotating disc in a transverse air stream, Thesis submitted to the University of Leicester, 1975.

[5] SACADURA J.F., Initiation aux transferts thermiques, Lavoisier, 1980.

[6] PAJANI, D., Mesure par thermographie infrarouge, ADD Editeur, 1989.

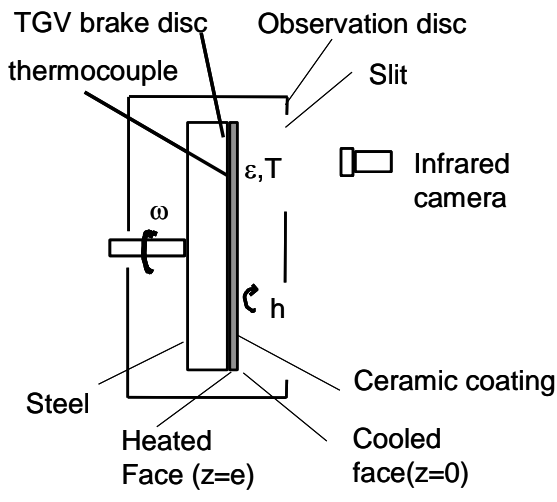

Figure 1: Principle of the method

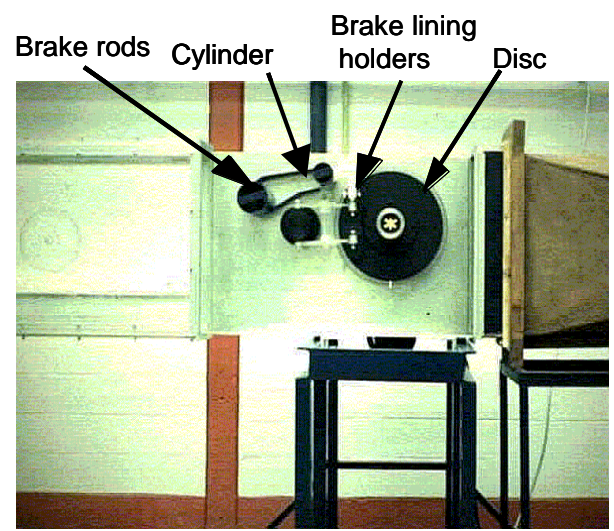

Figure 3 : Experimental set-up view

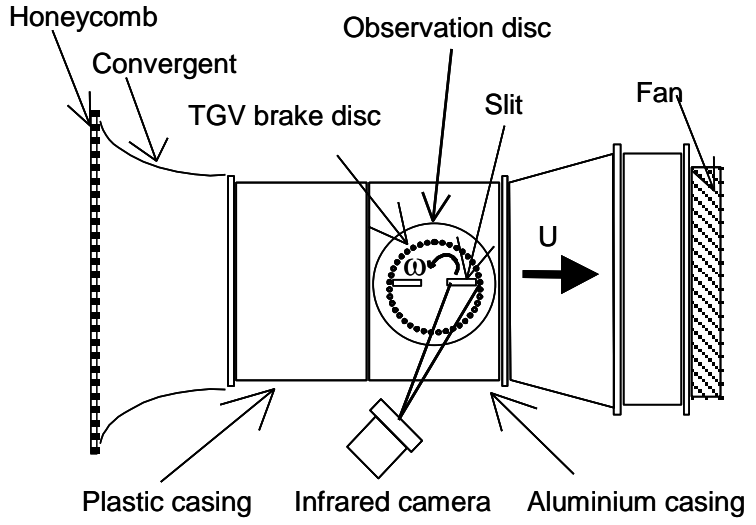

Figure 2: Experimental set-up

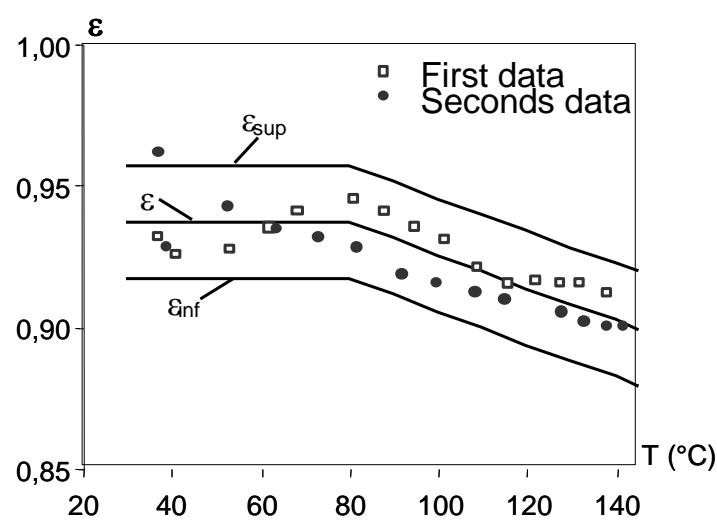

Figure 4 : Black paint emissivity 


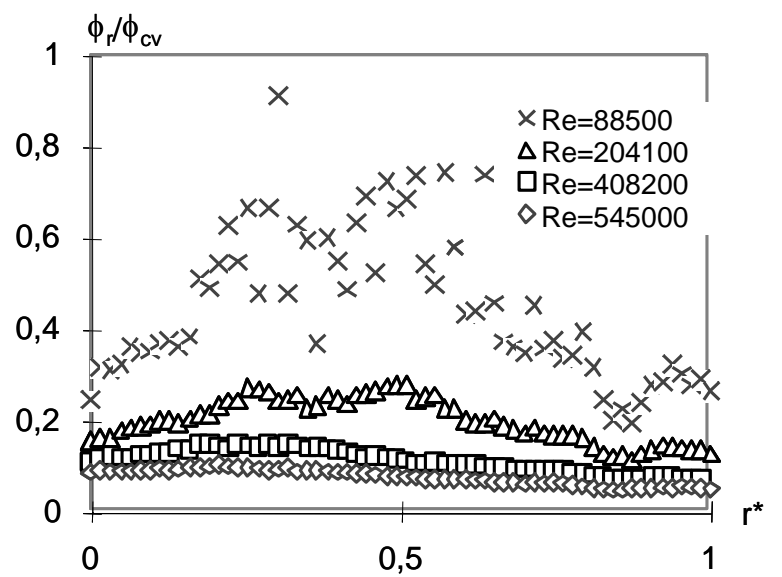

a) $\mathrm{Re}_{0}=0$

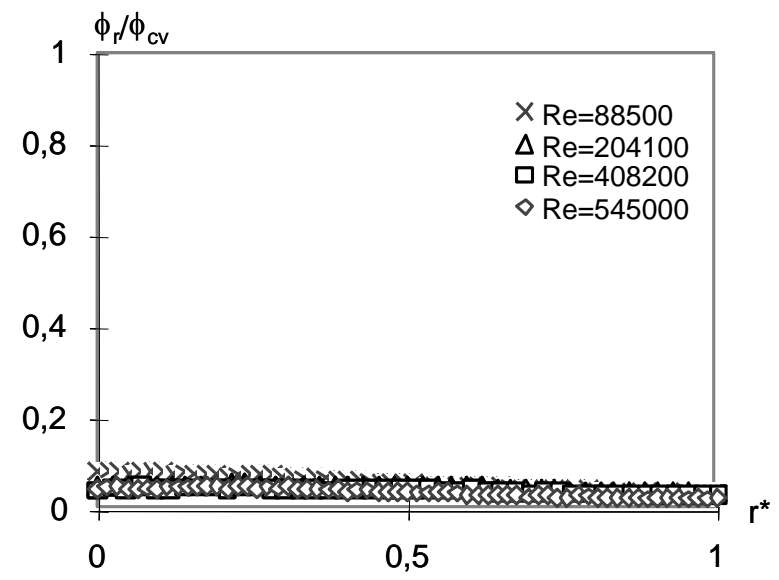

b) $\mathrm{Re}_{0}=153000$

Figure 5 : Contribution of the radiative losses compared to the convectives losses
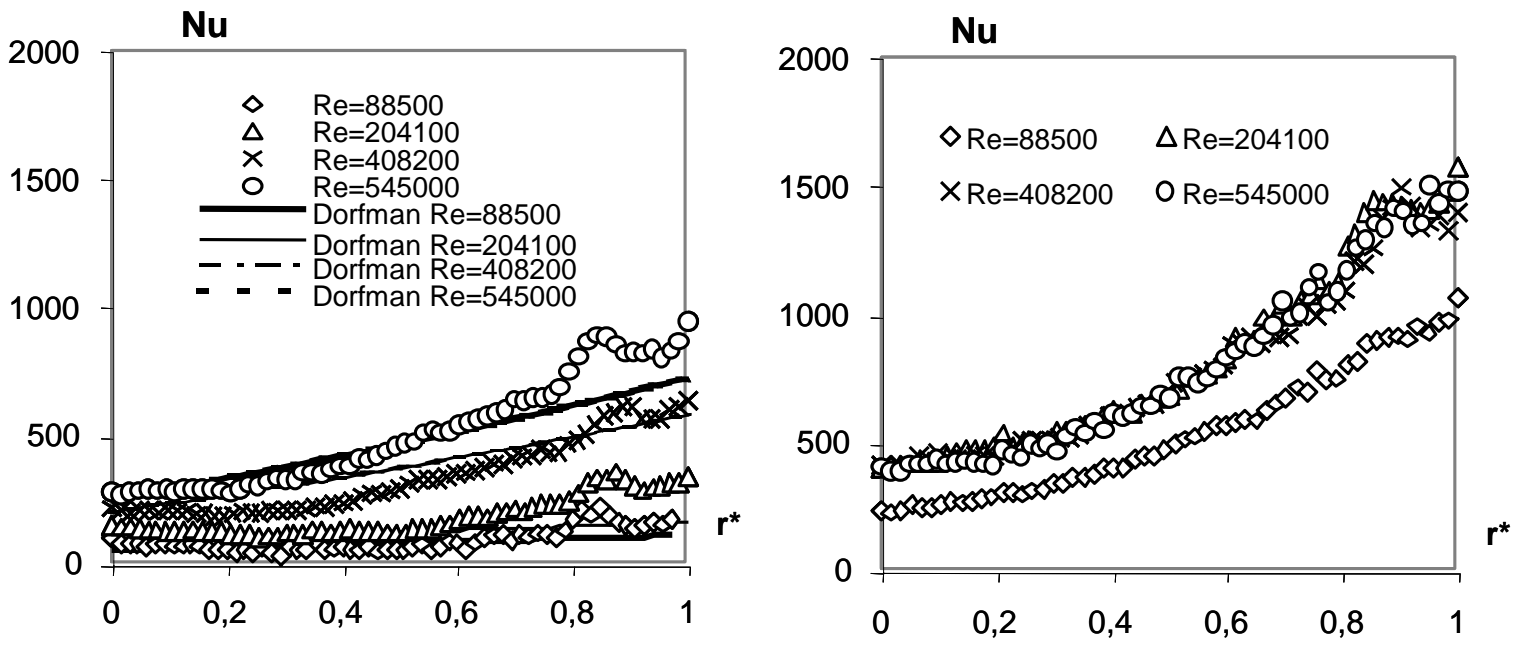

a) $\mathrm{Re}_{0}=0$

b) $\mathrm{Re}_{0}=153000$

Figure 6 : Variation of local Nusselt number as a function of dimensionless radius

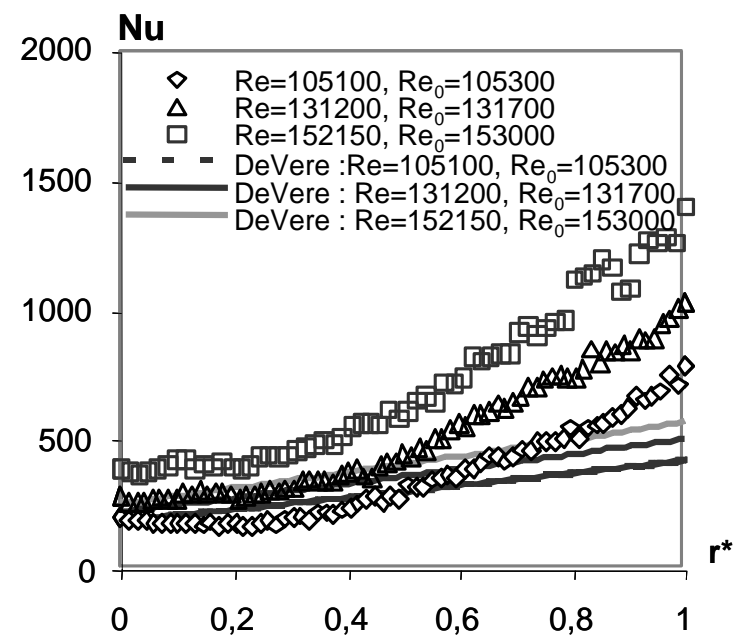

Figure 7 : Variation of local Nusselt number as a function of dimensionless radius for U=R. $\omega$ configuration 